\title{
Intraperitoneal chemotherapy in ovarian cancer treatment: are we missing something?
}

\author{
Interview with Pierluigi Benedetti Panici ${ }^{1}$ and Philipp Harter $^{2}$ by Claudia Marchetti ${ }^{1}$ and \\ Angela Musella ${ }^{1}$
}

In favor:

Pierluigi Benedetti Panici ${ }^{1}$

Against:

Philipp Harter ${ }^{2}$

\section{Can we consider intraperitoneal chemotherapy as a standard of care in advanced ovarian cancer treatment?}

\section{Pro intraperitoneal chemotherapy}

The rationale for administering chemotherapy directly into the peritoneal cavity is supported by preclinical, pharmacokinetic, and pharmacodynamics data. The most common route of ovarian cancer (OC) spread is within the peritoneal cavity. There are two potential explanations to clarify the efficacy of intraperitoneal (IP) chemotherapy. The first is the existence of the peritoneal-plasma barrier, a monolayer of mesothelial cells supported by a basement membrane and five layers of connective tissue. Due to this barrier, the IP delivery of anticancer agents may provide a pharmacokinetic gain by delivering drug levels that are 20 -fold to 1,000 -fold higher in the IP cavity than in plasma [1]. Second, the existence of cancer stem cells, which represent a subset of tumor cells efficient in self-renewal capacity [2]. In preclinical models, IP delivery of therapeutic agents has been shown to effectively eradicate OC stem cells [3-4]. In addition, three Gynecologic Oncology Group (GOG) multicenter, randomized, phase III clinical trials found IP chemotherapy to be superior to standard intravenous (IV) infusion in the primary medical management of small volume residual, advanced epithelial OC [5-7] (Table 1). Finally, in 2006, the National Cancer

'Department of Gynaecological and Obstetrical Sciences and Urological Sciences, Sapienza University of Rome, Italy. 2Department of Gynaecology and Gynaecological Oncology, Kliniken Essen Mitte, Essen, Germany.

Correspondence to: Claudia Marchetti, MD

Dipartimento di Scienze Ginecologiche-Ostetriche e Urologiche

Viale del Policlinico 155, 00161 Roma, Italy.

Phone: +39 064940550 - Fax: +3906 49972564

E-mail: clamarchetti81@gmail.com

CANCER BREAKING NEWS 2016;4(2):12-16

DOI: $10.19156 / \mathrm{cbn} .2016 .0013$
Institute (NCI) issued a rare Clinical Announcement encouraging the use of intraperitoneal and intravenous (IP/ IV) chemotherapy [8]. Therefore we can consider this approach as a possible standard treatment of advanced OC.

\section{Against intraperitoneal chemotherapy}

We can definitely not consider IP chemotherapy as a standard of care in advanced OC. After GOG 172 showed a non-significant difference in progression-free survival (PFS) and a significant difference in overall survival (OS) favouring IP chemotherapy, which stimulated multiple discussions about the toxicity of the studied regimen, we have now seen the results of GOG 252, another prospective randomized trial, which has shown clearly that there is no benefit in applying a chemotherapy intraperitoneally instead of intravenously.

\section{How should we interpret data coming} from randomized clinical trials about IP in the front line setting? Which have been the main concerns about the introduction of IP therapy in clinical practice?

\section{Pro the data showing a benefit}

As stated above, several randomized clinical trials have demonstrated that IP/IV chemotherapy improves survival in women with optimally resected, stage III OC, compared with IV chemotherapy alone [5-7]. A recent long-term survival analysis of the GOG 114 and 172 trials [9] showed that the median survival with IP therapy was 61.8 months ( $95 \%$ CI, 55.5 to 69.5 ), compared with 51.4 months with IV treatment. Despite these findings, there is a lack of consensus on whether IP therapy represents the best treatment for women with optimally cytoreduced epithelial OC. Possible reasons include the increased toxicity associated with this regimen with frequent discontinuations of treatment compared with standard IV administration of chemotherapy (in the GOG $172,42 \%$ and $83 \%$ completed planned IP/IV versus IV therapy, respectively [7]). Furthermore, regimens used in the experimental arms were different among studies, raising the question of whether the benefit associated with the IP administration was due to the type of schedule rather than the incorporation of IP treatment. However, as 


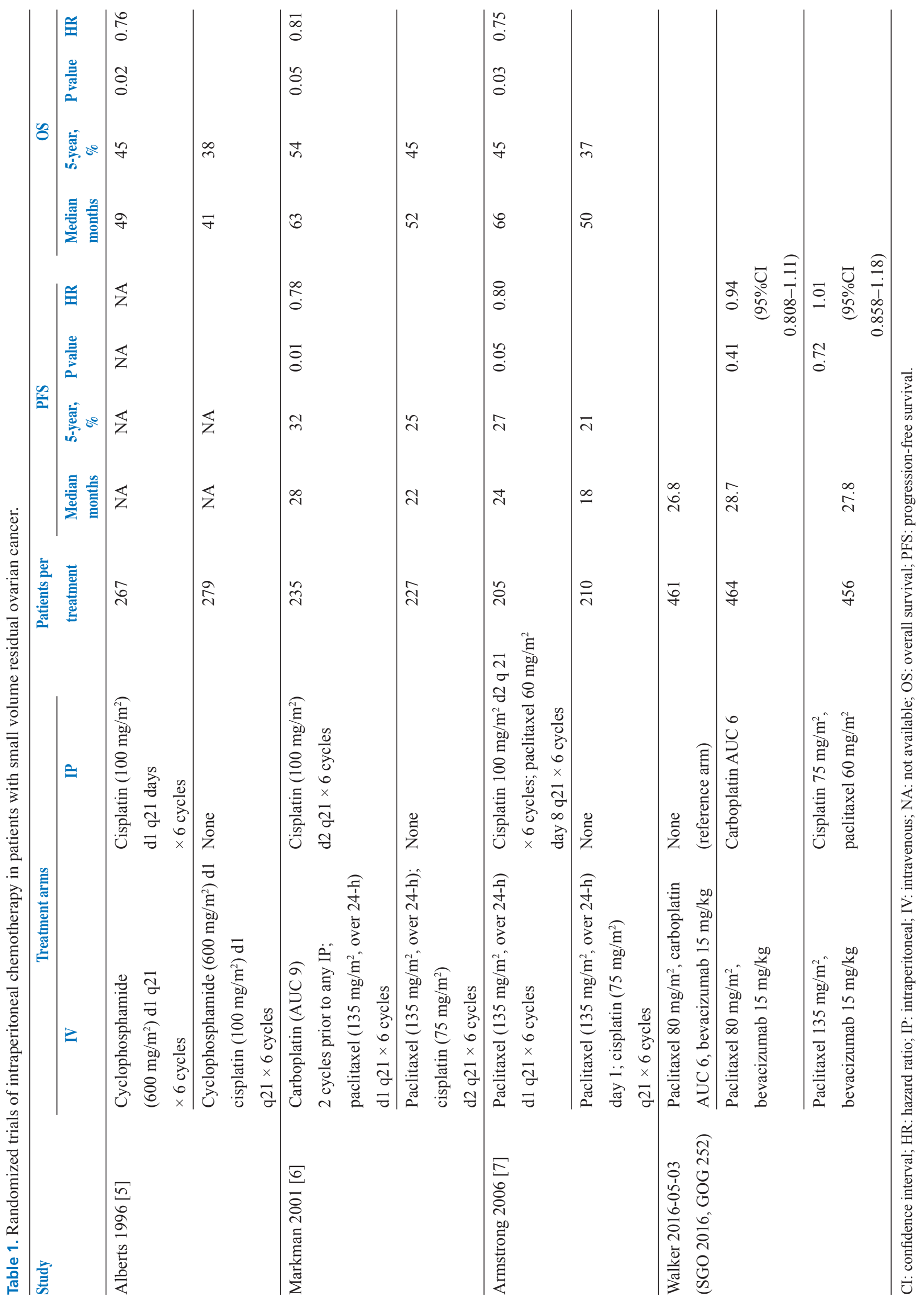


reported in our previous experience, the avoidance of additional surgery for the placement of the catheter by employing the direct puncture technique under ultrasonographic guidance can be expected to find most patients well-disposed to receive IP chemotherapy. Moreover, the majority of our patients experienced a mild pain level measured by visual analogue scale (VAS), achieving a high compliance rate $(94.7 \%)$ [10].

On the other hand, more recently data from the GOG 252 phase III trial [NCT00951496] have scaled down the role of IP therapy. Results showed a median PFS of approximately 27 to 29 months in patients with optimal stage II-III disease treated with regimens consisting of different combinations of IV and IP cisplatin, carboplatin, and paclitaxel, in combination with bevacizumab. An analysis limited to patients with optimal stage III tumors and no gross residual disease produced median PFS values of 31 to 34 months. These results were surprising, and in contrast to the results of the decade-old GOG 172 trial. Nevertheless, the results of this one trial should not outweigh the significant prior evidence that suggests that IP chemotherapy is beneficial. As far as we are aware, in the last 20 years IP has been the only approach able to significantly improve OS [5-7].

Until the results of ongoing trials are known, patients should be carefully counselled about this option, and IP chemotherapy should still be offered to women who are likely to be able to tolerate the significant toxicities associated with the treatment. Further randomized trials should be designed with the aim of identifying those who might benefit the most.

\section{Against the data showing a benefit}

As already pointed out, there were many discussions in the past about the balance between toxicity and potential benefit. Now, we have seen that there is not any benefit if we compare a possible IP chemo-regime with a modern IV regimen that includes antiangiogenic treatment.

\section{IP therapy has been shown to be} particularly effective in BRCA1/2 mutated patients. Should it be incorporated into the treatment algorithm in this setting of patients?

\section{Pro consideration as a treatment option}

BRCA status has become to be considered as a "marker" of better prognosis and platinum response [11]. Cisplatin induces intra- and inter-strand cross-links and activate apoptosis if the damaged DNA is not adequately repaired. The IP administration of cisplatin produces a 12-fold greater drug exposure in the peritoneal cavity. Therefore it has been speculated that patients with aberrant tumor expression of BRCA1/2 expression will have a diminished ability to repair double-stranded DNA breaks and might therefore be more sensitive to IP platinum-based therapy. Data testing this hypothesis are still scarce but some experiences have been particularly interesting and encouraging. In particular, Lesnock et al [12] evaluated BRCA1 protein expression via immunohistochemistry in tumor specimens from patients enrolled in GOG 172 with respect to PFS and OS. Interestingly, they found that nearly $50 \%$ of tumors had aberrant BRCA1 expression. Among women with aberrant BRCA1 expression there was a significant survival increase, with a median OS of 84 months, compared with 47 months in the IV group $(p=0.0002)$. In contrast, in tumors with normal BRCA1 expression, the median OS was 58 months for IP group vs 50 months for IV group $(\mathrm{p}=0.818)$. These findings warrant validation but, if confirmed, we might be able to select patient who might take the greatest advantage from IP therapy, sparing the side effects of IP therapy for the other group of patients who might not benefit (i.e., those without BRCA mutation).

\section{Impractical to use}

Unfortunately, the BRCA status of patients with OC is usually unknown at first diagnosis. Therefore, it is difficult to implement BRCA specific therapies within a few weeks after primary surgery.

\section{Does IP therapy have a possible role in the recurrent setting?}

\section{Pro consideration for use in recurrent OC}

To date, the standard treatment for recurrent $\mathrm{OC}$ is mainly based on IV chemotherapy. However, survival remains poor despite current standard therapy and it is necessary to develop novel treatment strategies. Secondline IP chemotherapy in the treatment of recurrent disease has been rarely investigated and there have been no published randomized trials on such investigations. All published relevant reports are observational studies and showed IP therapy to have activity in the recurrent setting. Recently a propensity score-matching study has been published [13], with the aim of analyzing the therapeutic efficacy of second-line IP chemotherapy in the treatment of recurrent epithelial OC. In accord with the results of the randomized trials of front-line IP chemotherapy, this analysis showed that second-line IP chemotherapy yields significantly longer PFS compared with standard second-line IV chemotherapy (4.9 vs 2.4 months, $\mathrm{p}<0.001$, for platinum-refractory/resistant 
disease, and 9.8 vs 6.9 months, $\mathrm{p}<0.001$, for platinumsensitive disease). In addition, the completion rate of receiving all six cycles of assigned IP chemotherapy was comparable between front-line and second-line chemotherapy. Taken together, implementation of second-line IP chemotherapy is worthy of consideration for patients who are diagnosed with recurrent epithelial ovarian, tubal and peritoneal cancer.

\section{Against use in recurrent disease}

There are no data from prospective randomized trials in this clinical setting. Therefore, this could not be recommended outside of clinical trials.

\section{What are the critical obstacles and the next challenges to improve the role of IP chemotherapy in OC treatment?}

\section{P. Benedetti Panici}

As previously mentioned, IP therapy has shown to be effective in front-line OC treatment. Nonetheless, several issues have limited the integration of IP/IV chemotherapy into practice, the most important concerning the high rate of complications caused by the IP catheter, from which follows low feasibility and compliance of IP therapy. In fact, the incidence of IP access device-related complications ranges between $7 \%$ and $34 \%$ with a consequently high discontinuation rate [10].

In the GOG 172 study, the discontinuation rate of $58 \%$ was due to catheter-related problems (34\%), of which $17 \%$ were catheter infections, $8.8 \%$ were blocked catheters, and $2.5 \%$ were leaking catheters [14]. Therefore, one of the main challenges is to find a different technique of IP administration. Previous studies reported successful IP access via direct injection using a peripheral venous catheter as a possible way to administer IP therapy. In a prospective pilot study we investigated the feasibility, complication rate, and compliance of the administration of IP chemotherapy by direct puncture under ultrasonographic guidance [10]. Among the 38 patients enrolled, with 402 IP procedures performed we found the feasibility rate was $97.4 \%$. We recorded a total of $2.25 \%$ mild intra-procedure complications. No significant difference was identified for the complication rate according to the abdominal wall thickness or according to the number of previous laparotomies. A total of 5 procedures $(1.2 \%)$ were not performed as a result of patients' noncompliance. The mean pain score according to the visual analogue pain scale was moderate at the first evaluation (after 3 minutes) and mild at the final evaluation (after 10 minutes). Therefore, we believe that an alternative route of administration such as direct puncture under ultrasound guidance might be a safe and feasible method, with a high acceptance from patients. Further studies are needed to confirm this data. Moreover, we are waiting for final results of the PETROC/OV21 trial, whose primary promising data have been recently presented at ASCO meeting. This trial has been designed to investigate the role of adjuvant IP chemotherapy in women not able to be optimally debulked at primary surgery and thus submitted to neoadjuvant IV chemotherapy and optimal interval debulking surgery followed by IV or IP chemotherapeutic administration of carboplatin/cisplatin and paclitaxel. The endpoints of the study include recruitment rate, completion of treatment and toxicity, and 9-month progression rate to select the preferred experimental arm for a phase III study. Data presented at the ASCO 2016 meeting reported a median PFS of 11.3 months for Arm 1 (IV paclitaxel $135 \mathrm{mg} / \mathrm{m}^{2}+$ IV carboplatin AUC 5/6 with d8 paclitaxel IV $60 \mathrm{mg} / \mathrm{m}^{2}$ q21d) and 12.5 months for Arm 3 (IV paclitaxel $135 \mathrm{mg} / \mathrm{m}^{2}$ + IP carboplatin AUC 5/6 and d8 IP paclitaxel $60 \mathrm{mg} / \mathrm{m}^{2}$ q21d) $(\mathrm{p}=0.27)$. Median OS was 38.1 months (Arm 1) and 59.3 months (Arm 3) $(\mathrm{p}=0.40)$ with a lower rate of adverse events $>$ grade 3 for IP administration $(23 \% v s$ $16 \%, p=0.24)$. Thus, the primary conclusions of the authors were that the IP carboplatin-based regimen, post neoadjuvant chemotherapy and debulking surgery, is well tolerated and associated with a lower 9-month progression rate compared to IV therapy [15].

Finally a better understanding of the biology of ovarian cancers is essential to improve the outcomes of patients and to find out those who might benefit the most from the IP procedure.

\section{P. Harter}

Many physicians do not like IP therapy and the data presented to date have clearly shown that IP therapy does not improve the outcome of the patients. The results from two further prospective trials (iPOCC and OV-21) are pending. Perhaps the results will show further supporting evidence.

\section{Acknowledgments}

The authors thank Ray Hill, an independent medical writer, who provided native English editing and journal styling on behalf of HPS. This editorial assistance was funded by PharmaMar, Spain.

\section{Conflicts of Interest}

The Authors declare there are no conflicts of interest in relation to this article. 


\section{References}

1. Lu Z, Wang J, Wientjes MG, Au JL. Intraperitoneal therapy for peritoneal cancer. Future Oncol 2010;6:1625-41.

2. Li L, Neaves WB. Normal stem cells and cancer stem cells: the niche matters. Cancer Res 2006;66:4553-7.

3. Casagrande F, Cocco E, Bellone S et al. Eradication of chemotherapy-resistant CD44+ human ovarian cancer stem cells in mice by intraperitoneal administration of Clostridium perfringens enterotoxin. Cancer 2011;117:5519-28.

4. Ponnurangam S, Mammen JM, Ramalingam S et al. Honokiol in combination with radiation targets Notch signaling to inhibit colon cancer stem cells. Mol Cancer Ther 2012;11:963-72.

5. Alberts DS, Liu PY, Hannigan EV et al. Intraperitoneal cisplatin plus intravenous cyclophosphamide versus intravenous cisplatin plus intravenous cyclophosphamide for stage III ovarian cancer. N Engl J Med 1996;335:1950-5.

6. Markman M, Bundy BN, Alberts DS et al. Phase III trial of standard-dose intravenous cisplatin plus paclitaxel versus moderately high-dose carboplatin followed by intravenous paclitaxel and intraperitoneal cisplatin in small volume stage III ovarian carcinoma: an intergroup study of the Gynecologic Oncology Group, Southwestern Oncology Group, and Eastern Cooperative Oncology Group. J Clin Oncol 2001;19:1001-7.

7. Armstrong DK, Bundy B, Wenzel L et al. Intraperitoneal cisplatin and paclitaxel in ovarian cancer. N Engl J Med 2006;354:34-43.

8. Trimble EL, Alvarez RD. Intraperitoneal chemotherapy and the NCI clinical announcement. Gynecol Oncol 2006;103 (2 Suppl 1):S18-9.

9. Tewari D, Java JJ, Salani R et al. Long-term survival advan- tage and prognostic factors associated with intraperitoneal chemotherapy treatment in advanced ovarian cancer: a Gynecologic Oncology Group Study. J Clin Oncol 2015; 33(13):1460-6.

10. Benedetti-Panici P, Perniola G, Marchetti C et al. Intraperitoneal chemotherapy by ultrasound-guided direct puncture in recurrent ovarian cancer: feasibility, compliance, and complications. Int J Gynecol Cancer 2012;22(6):1069-74.

11. Ledermann JA, Drew Y, Kristeleit RS. Homologous recombination deficiency and ovarian cancer. Eur J Cancer 2016;60:49-58.

12. Lesnock JL, Darcy KM, Tian C et al. BRCA1 expression and improved survival in ovarian cancer patients treated with intraperitoneal cisplatin and paclitaxel: a Gynecologic Oncology Group Study. Br J Cancer 2013;108(6):1231-7.

13. $\mathrm{Lu} \mathrm{CH}$, Chang $\mathrm{YH}$ et al. Task Force on Intraperitoneal Chemotherapy of Ovarian Cancer. Second-line intraperitoneal chemotherapy for recurrent epithelial ovarian, tubal and peritoneal cancer: A propensity score-matching study. Chemotherapy 2016;61(5):240-8.

14. Walker JL, Armstrong DK, Huang HQ et al. Intraperitoneal catheter outcomes in a phase III trial of intravenous versus intraperitoneal chemotherapy in optimal stage III ovarian and primary peritoneal cancer: a Gynecologic Oncology Group Study. Gynecol Oncol 2006;100:27-32.

15. Mackay H, Gallagher CJ, Parulekar WR et al. OV21/PETROC: A randomized Gynecologic Cancer Intergroup (GCIG) phase II study of intraperitoneal (IP) versus intravenous (IV) chemotherapy following neoadjuvant chemotherapy and optimal debulking surgery in epithelial ovarian cancer (EOC). J Clin Oncol 2016;34(suppl):abstr LBA5503. 